Cédric Vonesch, François Aguet, Jean-Luc Vonesch, and Michael Unser

\title{
An introduction to
} fluorescence microscopy

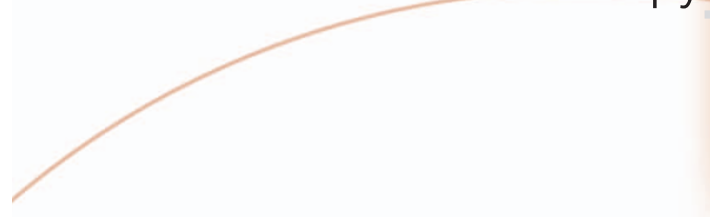

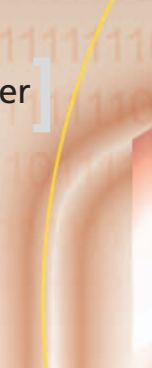

\section{The Colored Revolution of Bioimaging}

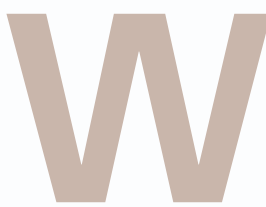

ith the recent development of fluorescent probes and new high-resolution microscopes, biological imaging has entered a new era and is presently having a profound impact on the way research is being conducted in the life sciences. Biologists have come to depend more and more on imaging. They can now visualize subcellular components and processes in vivo, both structurally and functionally. Observations can be made in two or three dimensions, at different wavelengths (spectroscopy), possibly with time-lapse imaging to investigate cellular dynamics.

The observation of many biological processes relies on the ability to identify and locate specific proteins within their cellular environment. Cells are mostly transparent in their natural state and the immense number of molecules that constitute them are optically indistinguishable from one another. This makes the identification of a particular protein a very complex task-akin to finding a needle in a haystack. However, if a bright marker were attached to the protein of interest, it could very precisely indicate its position. Much effort has gone into finding suitable markers for this purpose, but it is only over the course of the past decade, with the advent of fluorescent proteins, that this concept has been revolutionized. These biological markers have the crucial properties necessary for dynamic observations of living cells: they are essentially harmless to the organism, and can be attached to other proteins without impacting their function. 
Fluorescence microscopy was invented almost a century ago, when microscopists were experimenting with ultraviolet light to achieve higher resolutions. In the very beginning, observations were limited to specimens that naturally fluoresce. This property is called autofluorescence or primary fluorescence. Rapidly, fluorescent dyes for staining tissues and cells were investigated. But it was not until the 1940s that fluorescence microscopy became popular, when Coons and Kaplan introduced a technique to label antibodies with a fluorescent dye to study antibody-antigen interactions, profoundly changing the field of immunohistochemistry. The discovery that really brought fluorescence microscopy to the forefront came in 1994, when Chalfie et al. succeeded in expressing a naturally fluorescent protein, the now-famous green fluorescent protein (GFP), in living organisms. This was a landmark evolution in the field, fostering a whole new class of tagging methods.

While genetic engineering is at the origin of this new methodology, a number of innovations from the fields of physics, optics, and mechanical and electrical engineering have been combined to provide the necessary instrumentation. Impressive enhancements in classical microscopy have been achieved, and new imaging systems are actively being developed. A key element for the evolution of microscopy in general was the shift to digital imaging in the 1990s, with the availability of affordable high-sensitivity acquisition devices and powerful computer hardware.

The capabilities of today's systems often lead to enormous data sets that, in most cases, require postprocessing for their interpretation. Signal processing methods for biological research are only at their prelude; the needs are considerable and most probably not even clearly formulated yet. It is thus predictable that signal processing will be one of the main challenges of fluorescence microscopy in the forthcoming years.

The goal of this article is to provide an overview of the main aspects of modern fluorescence microscopy. We first cover the principles of fluorescence and highlight the key discoveries in the history of fluorescence microscopy. In subsequent sections, we present the optics of fluorescence microscopes and examine various types of detectors. Finally, we discuss the signal and image processing challenges in fluorescence microscopy and highlight some of the present developments and future trends in the field.

\section{FLUORESCENCE IN MOLECULAR AND CELLULAR BIOLOGY}

\section{THE PHYSICAL PRINCIPLES OF FLUORESCENCE}

\section{DEFINITION}

Fluorescence is a phenomenon by which a molecule, upon illumination at a specific wavelength, reemits light at another (typically longer) wavelength. A molecule that has the ability to fluoresce is called a fluorophore or fluorochrome. (Specifically, the former describes an atomic compound responsible for fluorescence, while the latter is a more general term for a dye that renders a body fluorescent.) It has distinctive excitation and emission spectra (see Figure 1), although in practice, it is often characterized by the two wavelengths corresponding to the respective peak intensities of these spectra.

A molecule can exist in a variety of energetic states, which, for the most part, are determined by the configuration of its electrons and the vibrational agitation of its atomic nuclei. If a photon with sufficient energy is absorbed by a fluorophore, the latter moves from its ground state to an excited electronic state [see Figure 2(a)]. Fluorescence occurs when the excited molecule returns to the ground state by releasing energy through emission of a photon. Because some of the energy gained during excitation is converted to heat, the emitted photon has a lower energy than the absorbed one. This explains the difference in wavelength mentioned earlier (since $E=h v=h c / \lambda$ ), which is also known as the Stokes shift. Fluorophores whose spectra present a large Stokes shift are usually preferred since their emitted light can be separated from the excitation light more easily by the means of filters (see Figure 1).

\section{RELATED PHENOMENA}

When in an excited state, a fluorophore can be forced to the ground state in a process called stimulated emission. Upon absorption of a second photon at the excitation wavelength, the molecule returns to the ground state by emitting two photons that are in phase and whose wavelengths are identical to the second photon's wavelength. This phenomenon is relevant to some of the concepts discussed later, but is best known as the lightamplification principle behind lasers.

Another important concept is that of multiphoton excitation. A fluorophore can also be excited by the simultaneous absorption of two or more photons, given that the combined energy of the photons corresponds to the energy required for single-photon excitation [see Figure 2(b)]. In this particular situation, the

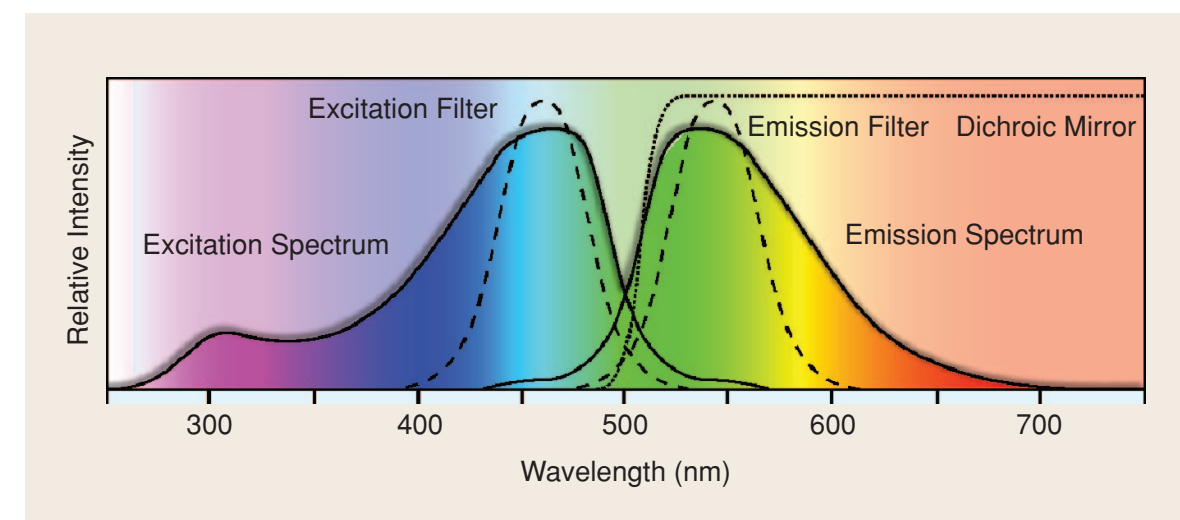

[FIG1] Representation of typical excitation/emission spectra of a fluorophore (in relative intensities). The excitation spectrum shows the emission intensity as a function of excitation wavelength, and the emission spectrum shows the relative emission intensity as a function of emission wavelength for an excitation at the peak absorption wavelength. 


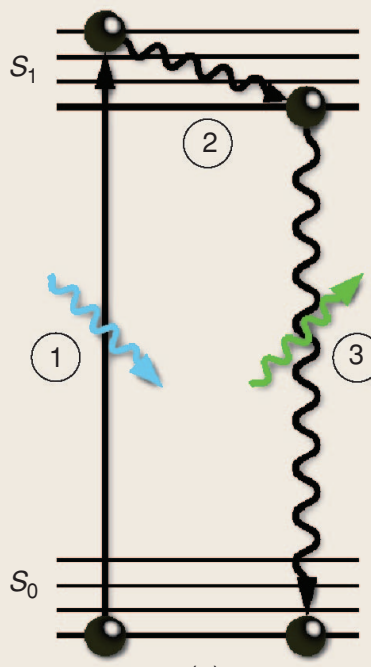

(a)

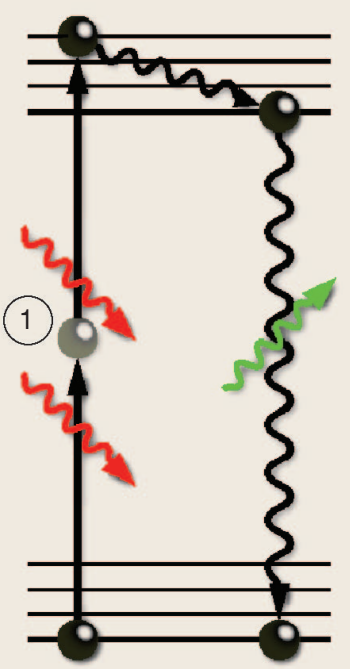

(b)
[FIG2] Jablonski diagrams representing the energy-level transitions involved in the fluorescence of GFP. Thick lines represent electronic energy levels; thin ones are associated vibrational energy levels. (a) Upon absorption of a photon at a specific wavelength (blue), the molecule moves from the ground state $S_{0}$ to the excited state $S_{1}(1)$. Vibrational energies are immediately converted into heat in a process called vibrational relaxation (2). When the molecule returns to the ground state, the remaining energy is released via emission of a new photon at a longer wavelength (green). (b) In the case of two-photon excitation, the excitation wavelength (red) is longer than the emission wavelength (green). The intermediate virtual state is indicated by (1).

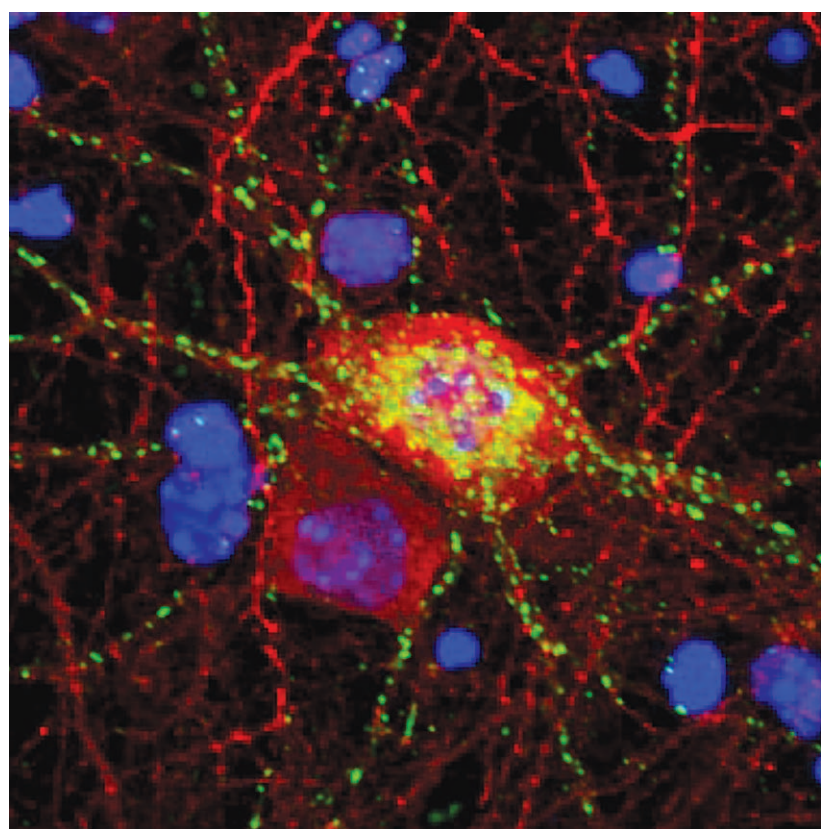

[FIG3] Image of a neuron where specific receptor proteins (delta opioid) have been fused with eGFP and appear in green. The red dots (GABA immunostaining) correspond to intracellular proteins located inside the neuron and its extensions. The nuclei of surrounding cells are stained in blue with DAPI, a fluorochrome that specifically binds to DNA. (Image courtesy of G. Scherrer, P. Tryoen-Toth, and B. L. Kieffer, IGBMC, Illkirch, France.) excitation wavelength is longer-in the case of two-photon excitation, twice as long as the single-photon excitation wavelength.

\section{THE GREEN REVOLUTION}

The developments that had the biggest impact on biological research and made fluorescence microscopy ubiquitous took place during the past two decades, and we shall thus focus on that period.

In the early 1990s, fluorescent labeling techniques such as immunofluorescence and covalent marking were already widely in use for imaging. Immunofluorescence is a technique (also called immunostaining) for detecting an antigen (protein) with a fluorochrome-labeled antibody. In covalent marking, proteins are purified, covalently labeled with a fluorescent molecule, and then introduced into cells. However, a straightforward means for selectively labeling a given protein with a nonperturbing fluorescent marker was not yet available. Only such a tag would make the in vivo observation of interactions between a specific protein with other proteins and the environment feasible.

The breakthrough came in 1994, when Chalfie et al. [4] succeeded in expressing a fluorescent protein that naturally occurs in a jellyfish species in other organisms by modifying their genome to code for this protein. At the origin of this innovation, accordingly dubbed "the green revolution" [35], was the discovery of GFP by Shimomura et al. in 1961 [33]. During their studies of the jellyfish aequorea victoria, whose fluorescing nature was described for the first time in 1955, they discovered that the source of the fluorescence was a naturally produced protein. Its chemical structure was reported by Shimomura in 1979, and in 1992, Prasher et al. cloned and determined its genetic sequence [30], paving the way for the work of Chalfie et al.

Since the first experiments with GFP, many variants have been engineered and discovered. From the naturally occurring GFP, called wtGFP for wild-type GFP, and from similar fluorescent proteins occurring in other marine organisms, new, more powerful mutants have been derived. Their properties range from different excitation and emission spectra to stronger fluorescence and higher resistance to photobleaching [38]. The currently available fluorescent protein tags offer a wide choice of wavelengths within the visible spectrum. Two widespread examples are cyan fluorescent protein (CFP) and yellow fluorescent protein (YFP), named for their characteristic emission spectra.

Biologists can label virtually any desired protein with a fluorescent protein by means of straightforward procedures. The first step leading to the creation of a labeled protein is to append the marker protein's sequence to that of the target. The resulting sequence is then introduced into cells, where its transcription results in the synthesis of a fusion protein. A common means for doing this is by placing the gene onto a plasmid - small, circular, double-stranded sequences of DNA that naturally occur in bacteria and are part of their genome - which can then be taken up by a cell. Inside the cell, they are expressed in the same fashion as chromosomal DNA. They are usually not replicated upon cellular division; however, in some cases they are integrated into the cell's chromosomal DNA. Plasmids exist for a wide range of fluorescent proteins and are available from specialized companies. 
The fusion protein (Figure 3) is expressed throughout the lifetime of the cell, as long as its sequence is present in the cell's nucleus. Note that this procedure typically results in the expression of both the fusion and natural versions of the protein, since the genetic sequence of the former does not replace that of the latter. Although the function and localization of the two variants are in most cases identical, it is necessary to verify that the label has no influence on cellular functions. For further details, we refer to [18] and [25].

The availability of fluorescent protein tagging techniques led to a fundamental change in the way biological research is conducted and to an explosion of experimental possibilities (see also the "Advanced Experimental Techniques" section). For instance, within the past ten years, the relative number of experiments dealing with live samples at the IGBMC imaging center (Illkirch, France) increased almost 100-fold.

\section{MICROSCOPES AND IMAGE FORMATION}

We now turn our attention to the instrumentation for fluorescence imaging. This section provides a brief description of the two main types of image forming systems: widefield and confocal microscopes. While the former are usually less expensive (depending on the configuration), their optical resolution is intrinsically more limited, especially in the axial (i.e., z) direction. Both systems can yield volume images of the sample under inspection, possibly with the help of deconvolution. However, in widefield microscopes the volume is acquired plane by plane (as opposed to point by point in standard confocal systems), which allows for faster acquisitions.

\section{THE WIDEFIELD MICROSCOPE}

\section{PRINCIPLE}

Widefield microscopy is based on the paradigm of Köhler illumination, according to which the sample is observed under a uniform light beam. Figure 4(a) shows how this is obtained in a simplified epi-illumination microscope: the light source (an arc or filament lamp) is magnified by the collector lens and projected onto the iris diaphragm. This aperture is located in a conjugate plane of the objective's back focal plane. Therefore, the latter acts as a condenser lens and the intensity from the iris is uniformly dispatched on the sample.

Let us now consider a single point of the sample. It will reemit light by reflection and possibly by fluorescence. If located in the focal plane, this will generate a beam of parallel light rays through the microscope tube. The image is formed by integrating the effect of all secondary point sources within the specimen; it can be observed through the eyepiece or recorded by placing a CCD sensor in the image plane.

One of the critical parameters in this setting is the numerical aperture (NA); that is, the angular opening of the light cone emerging from the object and collected by the objective. The magnification effect results from the combination of the objective, tube, and ocular lenses.

\section{COMPONENTS FOR FLUORESCENCE IMAGING}

Fluorescence imaging requires specific additional components for controlling the spectrum of the light (see also Figure 1). While typical lamps will produce "white light" (covering the whole visible spectrum, with some peaks at characteristic wavelengths), the fluorescent sample has to be illuminated with a specific excitation wavelength. This is ensured by inserting an excitation filter on the illumination path. The emission filter, on the other hand, ensures that only the wavelength corresponding to fluorescence reemission gets transmitted to the sensor or to the eyepiece, whereas reflected light (at the excitation wavelength) is discarded. A dichroic mirror helps achieve this by reflecting light below a certain transition wavelength (which is chosen to be between the excitation and emission wavelengths of the fluorophore) and transmitting light above that wavelength.

\section{INCOHERENT POINT SPREAD FUNCTION}

Because of the random nature of photon reemission, fluorescence microscopy is an incoherent imaging process. This means that each point of the sample contributes independently (without interference) to the light intensity distribution in the image space. Moreover, in the paraxial approximation, moving the object does not influence its image, aside from a shift. From a signal processing standpoint, a widefield microscope can thus be modeled in intensity as a linear space-invariant system. In other words, the light intensity (which is the physical value measured

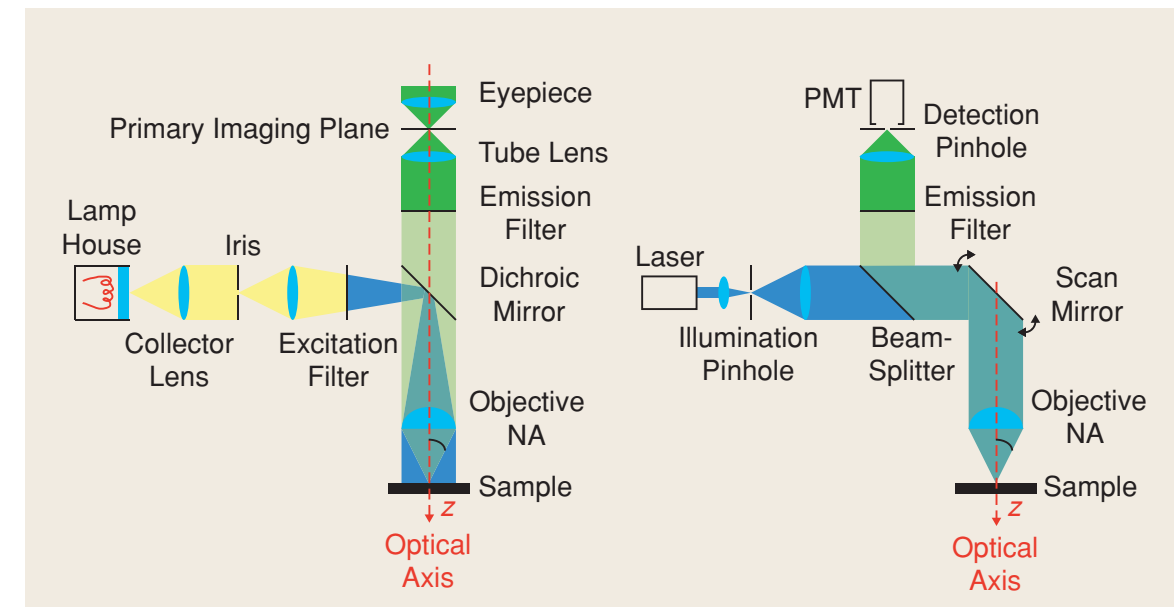

(a)

[FIG4] Schematics of (a) widefield and (b) confocal fluorescence microscopes, showing their main components. The illumination path is shown in yellow and/or blue (excitation at 395 $\mathrm{nm}$ ) and the image-forming path in green (emission at $509 \mathrm{~nm}$ ) to suggest the spectral composition of the different light beams in the case of a GFP-tagged sample. 
by a photodetector) in the neighborhood of the primary imaging plane $(z=0)$ is given by a convolutive expression

$$
\begin{aligned}
I(x, y, z) \propto & \int_{\mathrm{R}^{3}}\left|h_{\lambda_{\mathrm{em}}}\left(\frac{x}{M}-u, \frac{y}{M}-v, \frac{z}{M^{2}}-w\right)\right|^{2} \\
& \chi(u, v, w) d u d v d w
\end{aligned}
$$

where $M$ is the magnification of the objective (note that the axial magnification is $M^{2}$ ). Here, $\chi$ is the characteristic function of the object; it describes its ability to convert incident light into fluorescence intensity at the emission wavelength $\lambda_{\mathrm{em}}$ and is thus mostly related to the fluorophore concentration. The impulse response $\left|h_{\lambda_{\mathrm{em}}}\right|^{2}$ is called the incoherent (or intensity) point spread function (PSF), since it defines the image of an ideal point object $(\chi(x, y, z)=\delta(x, y, z))$. For a given wavelength $\lambda$, it is defined by a two-dimensional (2-D) Fourier transform

$$
\begin{aligned}
h_{\lambda}(x, y, z)= & \int_{\mathrm{R}^{2}} P(u, v) \exp \left(i 2 \pi z \frac{u^{2}+v^{2}}{2 \lambda f^{2}}\right) \\
& \exp \left(-i 2 \pi \frac{x u+y v}{\lambda f}\right) d u d v .
\end{aligned}
$$

In this expression, $f$ is the focal length of the objective. $P$ represents the pupil function, which is an indicator function that corresponds to the circular aperture of the objective. Its radius $r$ is related to the focal length by $\mathrm{NA} \simeq r / f$. Notice the presence of the depth coordinate $z$ in the phase factor; it accounts for the defocusing effect illustrated in Figure 5.

\section{THE CONFOCAL SCANNING MICROSCOPE}

\section{PRINCIPLE}

In a confocal microscope [23] [Figure 4(b)] the illuminating point source is usually obtained from a laser. The latter illuminates a pinhole located in a plane conjugate to the sample. In this way, the light is focused onto a very small volume of the sample, and the returning fluorescence radiation is collected by a photomultiplier tube (PMT) [26]. The essential difference with a widefield microscope is the detection pinhole, which drastically reduces the proportion of light coming from out-of-focus points, especially in the axial direction.

Since only one point is observed at a time, the object must be scanned. In the $x$ and $y$ dimensions, this is achieved by using a scan mirror, which deflects the illumination beam, hence moving the illumination spot in the same plane. In the $z$ direction, the sample is usually moved mechanically by the means of a motorized stage. The ability to resolve different planes within the object is called optical sectioning and leads to a complete volumetric representation (a stack of 2-D images).

A critical parameter in this setting is the pinhole diameter, which is usually expressed in Airy units (AU) (after back-projection in the object space, i.e., dividing the effective diameter by the magnification factor). One AU corresponds to the size of the central disc of the PSF of the system [Figure 5(b) center]. The smaller the pinhole, the better the resolution; however, this also means that less light is collected, implying a higher noise level.

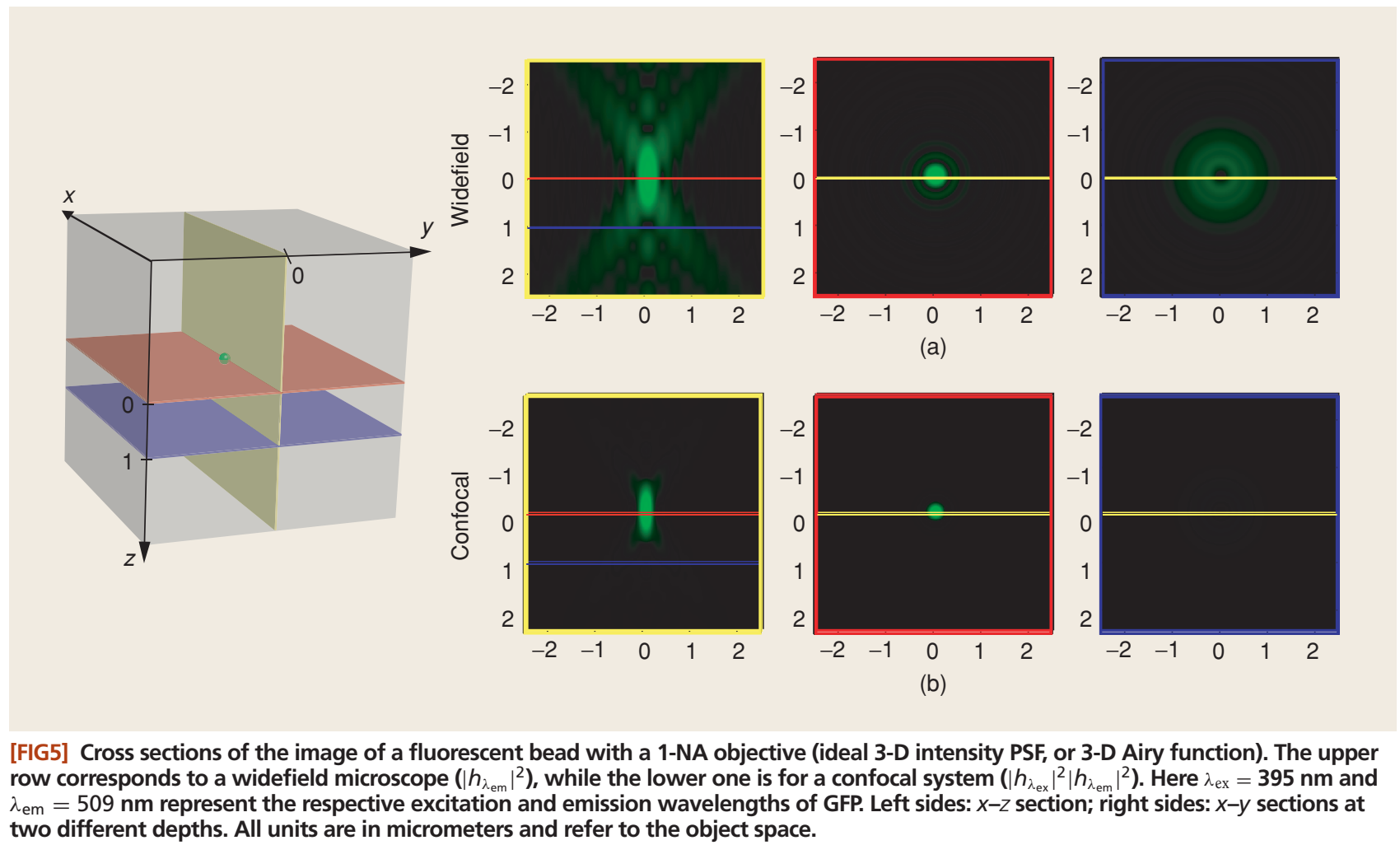




\section{INCOHERENT PSF}

The imaging process of fluorescent material can be modeled as follows: First, we have to take into account the effect of illumination, which consists of multiplying the fluorescence strength of the object by the PSF of the objective [taking into account the scan coordinate $\left.\left(x_{0}, y_{0}, z_{0}\right)\right]$. The reemitted light intensity is then given by

$$
\left|h_{\lambda_{\mathrm{ex}}}\left(x-x_{0}, y-y_{0}, z\right)\right|^{2} \chi\left(x, y, z-z_{0}\right)
$$

where $\lambda_{\text {ex }}$ denotes the excitation wavelength. The intensity at the detector is the convolution of this expression with the objective PSF, evaluated at the origin (the position of the detection pinhole)

$$
\begin{aligned}
\int_{\mathrm{R}^{3}}\left|h_{\lambda_{\mathrm{em}}}\left(x_{0}-x, y_{0}-y,-z\right)\right|^{2}\left|h_{\lambda_{\mathrm{ex}}}\left(x-x_{0}, y-y_{0}, z\right)\right|^{2} \\
\chi\left(x, y, z-z_{0}\right) d x d y d z
\end{aligned}
$$

where $\lambda_{\text {em }}$ denotes the reemission wavelength. Notice that we did not indicate the magnification factors here, which is equivalent to back-projecting the image into the object space. Also, the returning light beams are descanned when they hit back on the scanning mirror. Since $h_{\lambda_{\text {ex }}}$ is symmetric in $x$ and $y$, the final intensity PSF of the system is $\left|h_{\lambda_{\mathrm{ex}}} h_{\lambda_{\mathrm{em}}}\right|^{2}$, illustrated in the Figure 5(b). It appears that a confocal microscope has a PSF that is more concentrated in space than a widefield one, i.e., a better resolution, especially in the axial direction.

\section{SAMPLE SETUP AND ABERRATIONS}

In an ideal optical system, wavefronts propagate without undergoing phase distortions, also called aberrations. Modern microscope optics are highly sophisticated and are corrected to high levels of precision to avoid such distortions. The optical properties of the sample play an important role in the formation and correction of aberrations. Samples are usually placed onto a glass slide and need to be covered with a glass coverslip for use with most objectives. As shown in Figure 6, there is an immersion layer between the objective and the sample. (To increase resolution, an immersion medium with a high refractive index, such as oil, is used.) To minimize aberrations, each objective is designed for a specific setup, corresponding to parameters such as the refractive index of the immersion medium, the coverslip thickness, and the imaging depth. Small deviations from these optimal values (e.g., due to temperature changes or incorrect sample preparation) can introduce aberrations. A common and often unavoidable source of aberrations is the imaging depth in situations where the refractive indices of the specimen and immersion layers are mismatched. In the case where this mismatch is significant, it may result in the PSF becoming nonstationary, especially along the axial direction $z$ [9].

\section{LIMITING FACTORS OF FLUORESCENCE IMAGING}

Two sources act as the principal limiting factors in fluorescence imaging: 1) the instrumentation, which, apart from its inherent resolution limitation, introduces measurement noise, and 2) the sample itself, the optical properties and emission characteristics of which are often nonideal.

\section{NOISE SOURCES}

\section{PHOTON SHOT NOISE}

The fundamental limitation of any photodetector resides in the random nature of photon emission. The arrival of photons at the detector is well-described by a Poisson process whose (statistical) intensity is proportional to the (physical) intensity of the fluorescence signal.

\section{BACKGROUND NOISE}

The ambient radiation, especially in the infrared domain, can also be a significant source of noise; it often requires the use of additional filters at the detection stage.

\section{DARK CURRENT}

Among the numerous internal noise sources of the detector, thermal agitation is the most important. The higher the temperature, the higher the kinetic energy of the electrons. For semiconductor detectors, this results in so-called dark currents (which exist even in the absence of light), which tend to charge the photocapacitors when the integration time and/or the temperature are too high. For point detectors such as PMTs, thermal energy can trigger spontaneous electron emissions. Consequently, high-sensitivity detectors are very often cooled down to reduce thermal noise.

\section{AUXILIARY NOISE SOURCES}

For semiconductor devices, additional noise is generated at read-out time. In particular, the charge transfer in CMOS sensors is less efficient than in charge-coupled device (CCD) chips. Both technologies are subject to amplifier noise. For PMTs, there can be fluctuations in the internal gain of the unit, which also result in noise. Finally, any detector with digital output produces quantization noise.

\section{SAMPLE-DEPENDENT LIMITATIONS}

\section{PHOTOBLEACHING}

An important property of fluorophores is that they become more chemically reactive as they are being excited. Depending on the environment, they can undergo reactions that lead to permanent changes, by which the molecule loses its capability to fluoresce altogether or becomes nonabsorbent for the specified excitation wavelength. This effect, called photobleaching, limits

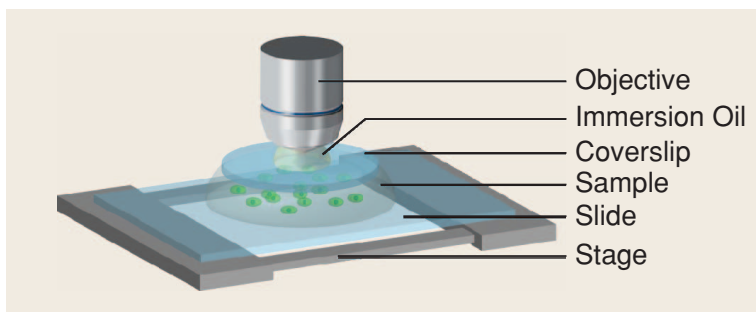

[FIG6] Schematic representation of a typical sample setup. 
the total intensity of light and, accordingly, the exposure time until loss of fluorescence occurs. As a result, the observation time of a fluorescence-tagged specimen is limited. Photobleaching is a cumulative effect, that is, reducing the exposure time or excitation intensity will not prevent it, but merely reduce the rate at which it occurs.

\section{AUTOFLUORESCENCE}

Many organic molecules are naturally fluorescent, and thus even unstained biological samples can emit fluorescence in the visible domain. This autofluorescence is an important source of noise when it overlaps with the emission of a selected fluorophore, especially when the latter is sparsely expressed or exhibits weak fluorescence. This interference can render the detection of a signal very difficult.

\section{ABSORPTION AND SCATTERING OF THE MEDIUM}

In a biological specimen, the intensity of the fluorescence signal decreases as the fluorophore's depth within the specimen increases. This attenuation is due to the absorption and scattering of light. Scattering is the phenomenon by which particles with a refractive index different from the medium's index partially diffuse electromagnetic radiation in all directions. It commonly occurs when the particle sizes are comparable to the wavelength. This attenuation strongly limits both the depth at which a fluorophore can be excited and the depth at which a fluorescence signal can be detected (typically in the $100-\mu \mathrm{m}$ range for one-photon confocal microscopy). These effects are not always negligible. Therefore, to obtain truly quantitative measurements, it may be necessary to develop reconstruction algorithms that take into account the space-varying and complex nature of the refractive index.

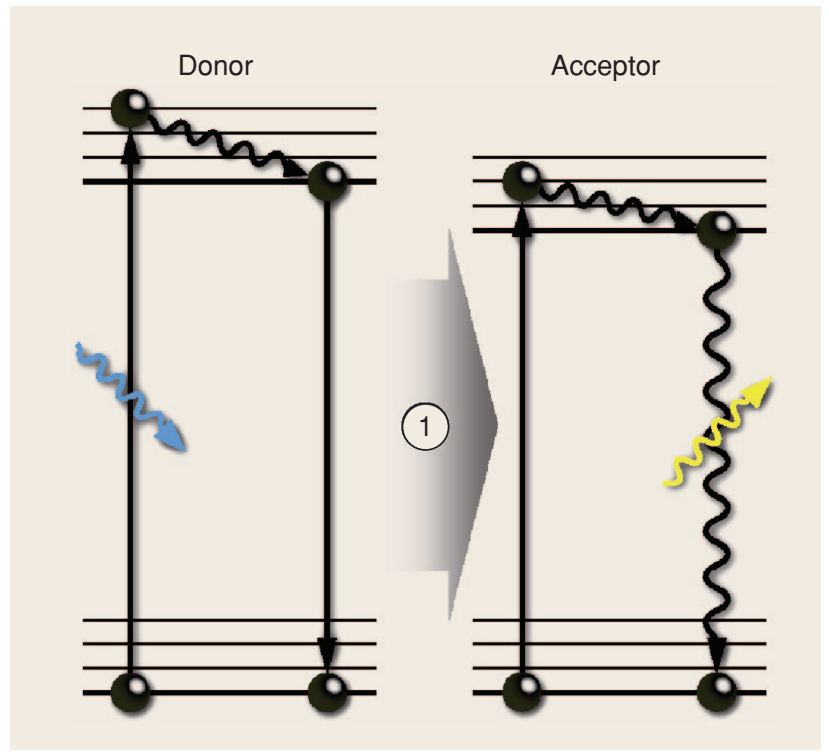

[FIG7] The principle of FRET between a suitable donor-acceptor pair: The energy of the excited donor molecule is transferred (without emission of a photon) to the acceptor after vibrational relaxation (1). For FRET to occur, the distance between the two molecules must typically be in the range of 1-10 $\mathrm{nm}$ [38].

\section{ADVANCED EXPERIMENTAL TECHNIQUES}

Besides standard imaging which involves the quantitative analysis of local fluorophore concentrations, there exist more sophisticated experimental techniques for studying protein-protein interactions and investigating biological processes at the molecular scale. Among the techniques presented below, fluorescence lifetime imaging microscopy (FLIM) and fluorescence resonance energy transfer (FRET) can be performed on both widefield and confocal microscopes. The photobleaching techniques, however, are usually performed with lasers and often require the ability to precisely define the region to be bleached; they are therefore mostly implemented on confocal microscopes.

\section{FRET}

Protein-protein interactions take place at scales that are too small to be resolved by optical microscopy; however, they can be detected by exploiting the mechanism known as the FRET phenomenon. It consists in a direct transfer of energy (i.e., it does not involve the emission or absorption of a photon) between a suitable donor and an acceptor, as illustrated in Figure 7. FRET is only possible between two fluorophores if the emission spectrum of the donor overlaps with the excitation spectrum of the acceptor. An example of a suitable pair of fluorescent proteins is the aforementioned CFP/YFP couple.

The efficiency of FRET strongly depends on the distance separating the two molecules (the rate is inversely proportional to the sixth power of the distance) and on the relative orientation of their dipole moments. This means that FRET can be used to study the optical subresolution colocalization of a labeled protein pair of interest. FRET can also serve as an indicator of conformational changes in a protein; if complementary markers are placed at the extremities of the protein, then an energy transfer can occur when the protein folds.

\section{FLUORESCENCE RECOVERY AFTER PHOTOBLEACHING}

Although photobleaching has already been mentioned as a limitation, it can be exploited to study the intracellular dynamics of proteins. Fluorescence recovery after photobleaching (FRAP) consists of intentionally bleaching a small region of a cell using high-intensity light, thereby rendering it nonfluorescent. The region then regains its fluorescence as fluorophores from the surroundings enter and pass through it, yielding information about the diffusion and mobility of the fluorophore.

\section{FLIM}

All of the techniques discussed up to this point rely on intensitybased measurements. In the presence of autofluorescence, or when multiple fluorophores with similar emission spectra are used, it can be difficult to discriminate among the different signals. Intensity-based imaging is also highly dependent on fluorophore concentration.

In FLIM, image contrast is generated based on the lifetime of fluorophores, which is the average time a fluorophore remains in the excited electronic state. The key point is that every 
fluorophore has a unique lifetime. A common method for measuring fluorescence lifetimes consists of exciting fluorophores with a picosecond pulsed laser source and recording the arrival times of the emitted photons with a high-speed photodetector.

The lifetime of a fluorophore is sensitive to many environmental factors, such as oxygen concentration, $\mathrm{pH}$, and calcium ion concentration. Thus, FLIM can also be used to obtain information about the local environment of a particular fluorophore.

\section{SIGNAL AND IMAGE PROCESSING CHALLENGES}

\section{DATA SIZE AND DIMENSIONALITY}

Modern research in biology requires quantitative experimental data. As a consequence, microscopes have developed into sophisticated digital image acquisition workstations capable of acquiring very large data sets of high dimensionality.

To get a better feeling of what is involved, consider an experiment monitored with a confocal microscope that requires the periodic (time-lapse) 3-D acquisition of a sample labeled with two fluorophores. This yields a five-dimensional (5-D) data set indexed by the space coordinates $x, y$, and $z$, the time $t$, and the wavelength parameter $\lambda$. Assuming that each image has a resolution of $1024 \times 1024$ and that 32 slices are acquired per volume every 20 minutes over $24 \mathrm{~h}$ with a 12 -b quantizer, the whole experiment results in nearly $7 \mathrm{~GB}$ of data. If a comparative analysis is performed, this figure must be multiplied by the total number of samples.

Studies involving comparable or even larger amounts of data are becoming commonplace. Even with today's performance level of computer hardware, the storage, extraction, manipulation, and representation of such data sets remain complex. One major challenge lies in the design of database systems and compression formats allowing for efficient retrieval and visualization (projections, 3-D rendering-see the example in Figure 8).

But, most importantly, signal processing is becoming an indispensable expertise for the analysis and understanding of quantitative biological experiments. In fact, it is increasingly considered part of the experimental protocol itself, as a way to infer the validity of a biological model.

Without claiming exhaustiveness, we give examples of current image processing problems in biology in five main categories: image preparation, image restoration, image registration, image segmentation, and quantitative image analysis.

\section{IMAGE PREPARATION}

\section{IMAGE CALIBRATION}

Calibration is an important step both for image analysis and visualization. It can involve various preprocessing tasks such as histogram equalization, inhomogeneous illumination compensation, background correction, or image rescaling. While these tasks may appear relatively simple, some of them can rely on advanced signal processing.

\section{IMAGE SIMPLIFICATION}

In some cases, biological structures are too complex to be processed directly and an image simplification step is required. To preserve the objects of interest, the operator can choose among the wide range of available tools (morphological operations, filtering, multiresolution structures, diffusion equations); application-specific solutions can also be envisaged.

\section{FEATURE DETECTION}

Biological images often present characteristic elements such as particles and filaments. The detection of these features may require the development of optimized filters [16], [32], as well as multiresolution methods [27]. Here, a challenging aspect is the shape variability observed in live-cell imaging.

Experimentalists should at least be aware of the aforementioned preparation operations; otherwise, they run the risk of a significant loss of information, thereby leading to questionable results at publication time. Algorithm designers, on the other hand, should put more effort into education and the development of user-friendly imaging software.

\section{RESTORATION}

Restoration encompasses the classical problems of denoising and deconvolution.

\section{DENOISING}

Simple methods such as median filtering often need adaptation; for example, a 3-D stack may exhibit lower signal-to-noise (SNR) levels as deeper portions of the object are imaged, due to absorption and/or autofluorescence. More generally, restoration methods should be based on physically realistic noise models (e.g., Poisson statistics) and take into account various noise sources (see the "Limiting Factors of Fluorescence Imaging" section). Advanced algorithms relying on wavelet-domain thresholding strategies [6], PDE and variational formulations, or statistical frameworks are just starting to be used in the field of bioimaging and deserve more exploration.

\section{DECONVOLUTION}

This operation requires an adequate characterization of the

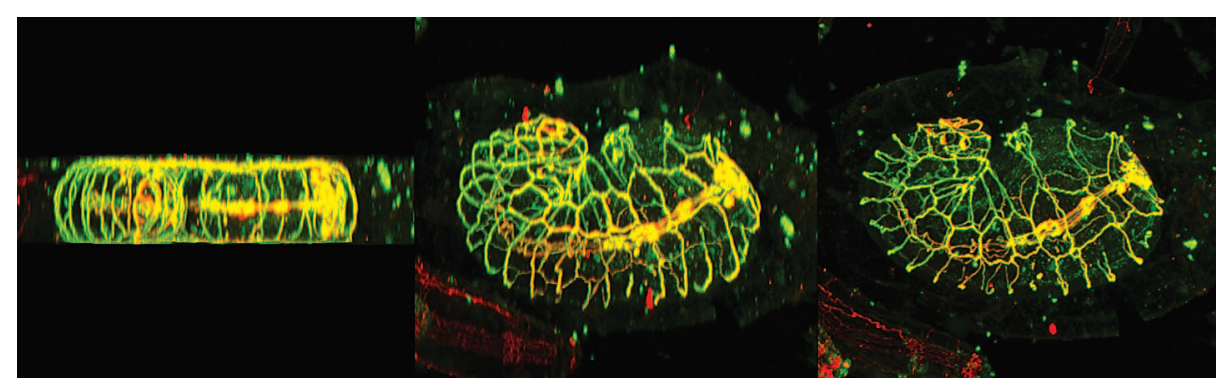

[FIG8] 3-D projections of a nematode worm embryo (C. elegans) [20]. (Image courtesy of L. McMahon, J.-L. Vonesch and M. Labouesse, IGBMC, Illkirch, France.) 
underlying imaging system, which can be either theoretical (involving a PSF model) or, frequently, experimental. In the latter case, the PSF is obtained by imaging subresolution fluorescent beads, under conditions as close as possible to the actual biological preparation. By averaging, possibly with simplifying assumptions (e.g., symmetry), a relatively noise-free PSF can be obtained. For further details concerning algorithmic deconvolution methods, we refer to the article by Sarder et al. [41].

One of the main challenges is the design of computationally tractable methods that take into account the nonstationarity of the PSF, especially in the axial direction (see the "Sample Setup and Aberrations" section). A recent attempt is the EM-algorithm proposed by Preza and Conchello [31]. In their image-formation model, the object is divided into several layers that are associated with a series of depth-dependent PSFs. The image of each layer is then obtained from classical (stationary) convolutions.

\section{OTHER INVERSE PROBLEMS}

Restoration can also be considered in the wider framework of inverse problems.

One example concerns relatively thick objects with surface labeling, observed under a widefield microscope. Because of the 3-D conical extension of its PSF [Figure 5(a), left], such a system has a limited depth-of-field; that is, only a small slice of the object around the focal plane appears sharp. To compensate for this, images at different focal depths can be taken and fused together so as to obtain a single, entirely sharp image. (This process should not be confused with deconvolution, which in particular yields a 3-D stack instead of a single image.) A stateof-the-art (so-called extended-depth-of-field) algorithm is described in [8]. Such a method can also be used to extract 3-D maps of the object's surface.

Another problem of interest is related to the detection and localization of subresolution particles [36]. New methods are under development that take into account the 3-D nonstationarity of the PSF to achieve precision in the nanometer range [1].

\section{REGISTRATION}

Registration is a frequently needed postacquisition step. Here, researchers can take advantage of the availability of high-quality registration algorithms that were initially developed for medical imaging.

\section{MOSAICING}

Because of the limited field of view of high-magnification objectives, it can be necessary to acquire multiple images of a sample, for example, in a mosaic scheme. Despite the high accuracy that sample stages can achieve, perfect alignment is never possible. Rigid-body registration algorithms can correct this, provided the acquired images or volumes slightly overlap. Within a given stack, it might also be necessary to compensate for pixel shifts between successive images. In addition, refractive indices, and thus focusing depths, are wavelength-dependent, which can necessitate the realignment of the different fluorescence channels.

\section{IMAGING LIVE SAMPLES}

During time-lapse acquisitions, spatial drifts can occur due to thermal processes; the sample itself might also be subject to motion. Therefore, even if the parts of interest lie in a relatively thin slice, they may not be observable in a unique focal plane over the whole experiment. This implies that either a stack of neighboring planes must be acquired and the planes of interest must be extracted (or fused) or a real-time focusing algorithm must be used to control the stage.

More sophisticated elastic registration may be required for compensating the deformation of living tissues or for matching specimens of comparable shape [34].

If a periodic biological process is too fast to be imaged with sufficient time resolution (such as the repetitive 3-D flow of blood cells in a heart), a registration approach may also be applied. In [17], images over several periods are recorded and reassembled so as to obtain a single period at a high frame rate.

\section{SEGMENTATION}

Segmentation is a mandatory step for image analysis. User interaction for the manual delineation of regions of interest is time consuming and lacks reproducibility. The need for automated segmentation methods is therefore important, e.g., for local intensity measures, object and event counting, and tracking.

While simple approaches such as prefiltering and thresholding are available in commercial software packages, advanced techniques, such as active contours [39], have not yet been much exploited in the context of biological image analysis.

The most accurate segmentation methods are often applicationdependent and typically require specific developments. For example, the tracing of neuronal dendrites can be improved using graph-optimization techniques [21].

In the context of live microscopy, it also makes good sense to adapt the segmentation methods so that they exploit temporal coherence, e.g., for the labeling of cells.

\section{QUANTITATIVE ANALYSIS}

\section{DATA PREPROCESSING}

In multispectral imaging, each pixel consists of (possibly a large number of) intensity measures at different wavelengths (obtained using different filter sets, an interferometer, or a diffractive system). If several fluorophores are used, their spectra are likely to overlap, and channel crosstalk must be expected. This gives raise to unmixing problems [40] that can be solved by taking separate reference images of each fluorophore (to measure its contribution to each channel) and using, for example, a singular-value decomposition [37]. Blind separation methods may also be applicable. As a general observation, the correct normalization of spectral data is critical for the interpretation of fluorescence images. Quantitative assessments using FRET or ratio imaging (comparing the relative intensities of different wavelengths) require careful preprocessing based on physical parameters such as spectrum overlap or fluorophore concentration. 


\section{MODEL FITTING}

Other advanced fluorescence techniques are based on the fitting of parametric models. In FLIM, the fluorescence lifetimes are obtained by fitting (possibly multiple) exponential trends to the photon arrival densities. In FRAP, the diffusion coefficients characterize fluorescence recovery curves [3]. Generally speaking, quantitative research often relies on the mapping of physical or biochemical models in the image and/or time domains, especially for dynamic processes.

\section{MOTION ASSESSMENT AND TRACKING}

The diffusion of fluorescent proteins can be characterized by estimating motion fields. In many instances, it is interesting to track individual objects, which can also be a challenging task. Two other articles in this issue cover the broad field of movement analysis, Meijering et al. [42] and Zimmer et al. [43].

PATTERN RECOGNITION AND CLASSIFICATION, SCREENING Screening experiments consist of a systematic, automated study of a large number of samples (up to several hundreds of thousands), e.g., for the study of gene function or for drug discovery. This can involve terabytes of data and several weeks of computerized analysis. Pattern recognition and classification algorithms play a major role in this analysis. In particular, one must identify how the biological characteristics of interest translate into measurable image features. Computational complexity is a strong limiting factor, while the reliability of the methods must be thoroughly validated. The article [44] gives more details on this topic.

\section{CURRENT AND FUTURE TRENDS}

In addition to the signal processing tools that have been discussed in the previous section, both the probes [38] and the instrumentation are being refined constantly. We therefore close our discussion with a description of current trends and future directions in the field.

\section{FLUORESCENT LABELS}

\section{QUANTUM DOTS}

Among the most recent developments are quantum dots [22], labels composed of a nanometer-sized semiconductor crystal core and an external protective shell. Their main advantages with respect to earlier fluorophores are their broader absorption and narrower emission spectra, resulting in brighter fluorescence. Also, they are more stable chemically and thus less subject to bleaching. These inorganic structures can be used for in vivo imaging, although they cannot be expressed by cells.

\section{LABELING OF RECOMBINANT PROTEINS}

The principle of this technique is to create fusion proteins that are not fluorescent by themselves, but which express a receptor to which a specific label can be added at a later time [10]. The label can be chosen from a wide range of fluorophores, with properties that GFP-type proteins may not be able to provide (such as higher resistance to photobleaching and stronger fluorescence). The receptor continues to be expressed in newly synthesized proteins, but only the stained proteins exhibit fluorescence, which allows for the selective labeling of a protein population at a given point in time.

\section{ENHANCED FLUORESCENT PROTEINS}

New fluorescent proteins are being developed that provide increased quantum efficiency (e.g., enhanced GFP, or eGFP, with a 35-fold increase in brightness with respect to the original GFP) or whose emission spectra are closer to infrared wavelengths (700 $\mathrm{nm}$ and above). These wavelengths are generally less absorbed by biological samples, hence allowing deeper observation. They are also less masked by cell autofluorescence occurring in the visible spectrum.

\section{PHOTOCONTROLLABLE PROTEINS}

Recent research has also been devoted to the design of photoactivatable [28] and photoswitchable [19], [2] proteins. The former exhibit little fluorescence in their initial, quiescent state. When exposed to a strong irradiation at a specific wavelength (usually lower than the fluorescence excitation wavelength), a 100-fold or higher increase in fluorescence brightness can be observed. For switchable proteins, strong irradiation changes both the excitation and emission spectrum. For example, PS-CFP [5] is sensitive to irradiation at $405 \mathrm{~nm}$, which produces a 1,500-fold increase in its green-tocyan ratio. Both types of labels can be used to activate and observe proteins in a specific region of a cell, without the interference of newly synthesized proteins or proteins outside of the selected region. This property is useful for protein lifetime and tracking as well as cell lineage studies.

\section{ADVANCED MICROSCOPY SYSTEMS}

We conclude this section with some of the more advanced developments in the field of optics.

FASTER SCANNING - SLIT DETECTORS AND NIPKOW-DISKS To cope with the high speed of some biological processes, the traditional confocal scanning microscope equipped with a point detector is often not sufficient. To accelerate the scanning process, a whole line can be imaged simultaneously by replacing the pinholes and the PMT by slit apertures and a linear camera. More generally, using e.g., a Nipkow-disk system [29], a 2 -D illumination pattern can be shifted across the sample, allowing time lapse imaging at up to 120 frames/s. This comes with a significant tradeoff in terms of resolution, due to crosstalk between the different detection apertures.

\section{DEEPER IMAGING—MULTIPHOTON MICROSCOPY}

In a multiphoton microscope [7], optical sectioning is achieved by properties of the illumination; as a consequence, there is no need for a detection pinhole in such a system. Very short laser pulses (in the pico- to femtosecond range) are sent to the sample in brief intervals (on the order of nanoseconds). The probability 
that two photons encounter the same molecule, hence bringing it to its excited state and making it fluoresce, is significant only in the very central region of the illumination spot. A key advantage is that the corresponding infrared wavelengths are less absorbed by biological tissues so that samples can be imaged much deeper than with traditional confocal systems (at a comparable resolution). Photobleaching and toxicity are also reduced because the excitation intensity is effectively concentrated at the focal spot.

\section{BETTER RESOLUTION-MULTIPLE OBJECTIVE IMAGING}

Since the numerical aperture has such a fundamental influence on resolution, Hell et al. proposed to insert the sample between two objectives, so as to send and collect light from both sides; accordingly, they called the method $4 \pi$ microscopy [13]. Using computational methods, an improvement in axial resolution by a factor of six can be achieved. Such systems are commercially available, but they suffer from limitations on the sample thickness and sensitivity to differences in the length of the two optical paths. More recently, these ideas have been applied to widefield microscopy ( $\left.\mathrm{I}^{5} \mathrm{M},[11]\right)$.

\section{OTHER DEVELOPMENTS}

Another promising technique to improve the resolution of widefield systems is structured illumination. Illuminating the object with sinusoidal patterns, combined with adequate processing, can result in a two-fold or higher [12] improvement of the microscope's spatial bandwidth. It also yields optical sectioning properties, and the processing can be done on specific hardware for real-time observation [24].

Another approach proposed by Stelzer et al. (selective plane illumination microscopy (SPIM), [15]) consists of sending the light on the object perpendicularly to the optical axis, in a diffraction-limited plane; then only fluorescence from molecules within this excitation plane is collected using a traditional CCD sensor. This system provides true optical sectioning for widefield systems.

Finally, one of the most advanced techniques is stimulated emission depletion (STED) [14]. The principle is to prevent fluorescent molecules outside the very central region of the illumination spot from emitting light by forcing them back to their fundamental state. This is achieved by dividing the excitation into two brief successive laser pulses, the second one having zero intensity at the center of the first one.

\section{CONCLUSIONS}

Although this panorama is necessarily incomplete, we hope to have convinced the reader of the invaluable role of fluorescence microscopy in modern biology. It owes its current popularity to the GFP-like fluorescent proteins that are the key ingredient for in vivo studies of molecular processes in cells. These are currently opening up a plethora of experimental possibilities that only begin to be explored.

This colored revolution could clearly not have happened without numerous technological advances. In particular, progress in optics and instrumentation has been considerable in recent years. There is now a consistent trend towards nonlinear techniques, such as multiphoton and saturated illumination imaging, which, with the help of computational methods, are contributing to overcoming Abbe's resolution barrier.

Signal processing is also at the heart of these developments and is expected to play an ever-increasing role in the field. It is already an integral part of optics and is becoming an essential tool for biologists, who rely more and more on imaging software to quantitate their data.

Therefore, a crucial aspect of the research lies in the successful collaboration between signal processing engineers and biologists. In modern research institutes, imaging core facilities are expected to play an important mediating role in this interaction. Our advice to colleagues that want to be part of this effort is that they try to understand the physics and, to some extent, the biology in order to design better and more useful algorithms. We believe that it is truly worth the effort.

\section{ACKNOWLEDGMENTS}

The authors thankfully acknowledge the helpful discussions with the following researchers in optics: C.J.R. Sheppard, E. Charbon, C. Depeursinge, and T. Lasser. They also thank Michel Aguet, Lucia Baldi-Unser, and Nathalie Garin for their advice on biological matters and their comments on the manuscript. The micrographs were kindly provided by the research groups of A. Giangrande, B.L. Kieffer, and M. Labouesse, all at IGBMC, Unité Mixte de Recherches CNRS/INSERM/Université Louis Pasteur, Illkirch, France. This work was funded in part by the Hasler Foundation.

\section{AUTHORS}

Cédric Vonesch (cedric.vonesch@epfl.ch) spent one year studying at the Ecole Nationale Supérieure des Télécommunications in Paris and graduated from EPFL, the Swiss Federal Institute of Technology in Lausanne, Switzerland, in 2004. He is now with the Biomedical Imaging Group at EPFL, where he is working on his Ph.D. thesis. His research interests include multiresolution and wavelet analysis, inverse problems, optics, and applications in bioimaging.

François Aguet (francois.aguet@epfl.ch) obtained his M.E. degree in computer science in 2004 from EPFL (Ecole Polytechnique Fédérale de Lausanne) in Lausanne, Switzerland. Currently, he is a Ph.D. candidate with the Biomedical Imaging Group at EPFL. His research interests include image processing, optics, and inverse problems in biomedical imaging.

Jean-Luc Vonesch (jlv@igbmc.u-strasbg.fr) is a specialist in image processing. In 1992, he created the optical imaging center at IGBMC ( 600 people, 40 research groups) and he has been leading it since. He is the originator of numerous tools, including a novel computer language for image processing. He has also proposed and developed a new fluorescence macroscopy system, which is now available as a commercial product. He regularly works as a consultant for leading manufacturers of microscopes and scientific instruments. He is also a lecturer in bioimaging at the Université Louis Pasteur, Strasbourg. He has contributed to more than 50 reviewed publications and was 
awarded a prize for innovation by INSERM (the French national institute for medical research) in 2003.

Michael Unser (michael.unser@epfl.ch) received the M.S. (summa cum laude) and Ph.D. degrees in electrical engineering in 1981 and 1984, respectively, from the Ecole Polytechnique Fédérale de Lausanne (EPFL), Switzerland. From 1985-1997, he worked as a scientist with the National Institutes of Health, Bethesda, Maryland. He is now a professor and director of the Biomedical Imaging Group at the EPFL. He is the associate editor-in-chief of IEEE Transactions on Medical Imaging and editor-in-chief of Wavelet Digest. He organized the first IEEE International Symposium on Biomedical Imaging (ISBI'2002) and currently chairs the technical committee of the IEEE-SP Society on Bio Imaging and Signal Processing (BISP). His main research areas are biomedical image processing, splines, and wavelets. He is a Fellow of the IEEE and the recipient of three Best Paper Awards from the IEEE Signal Processing Society.

\section{REFERENCES}

[1] F. Aguet, D. Van De Ville, and M. Unser, "A maximum-likelihood formalism for sub-resolution axial localization of fluorescent nanoparticles," Optics Express, vol. 13, no. 26, pp. 10503-10522, 2005.

[2] R. Ando, H. Hama, M. Yamamoto-Hino, H. Mizuno, and A. Miyawaki, "An optical marker based on the UV-induced green-to-red photoconversion of a fluorescent protein," Proc. Nat. Acad. Sciences, vol. 99, no. 20, pp. 12,657-12,662, 2002.

[3] G. Carrero, D. McDonald, E. Crawford, G. de Vries, and M.J. Hendzel, "Using FRAP and mathematical modeling to determine the in vivo kinetics of nuclear proteins," Methods, vol. 29, no. 1, pp. 14-28, 2003.

[4] M. Chalfie, Y. Tu, G. Euskirchen, W. Ward, and D.C. Prasher, "Green fluorescent protein as a marker for gene expression," Science, vol. 263, no. 5148, pp. 802-805, 1994.

[5] D.M. Chudakov, V.V. Verkhusha, D.B. Staroverov, E.A. Souslova, S. Lukyanov, and K.A. Lukyanov, "Photoswitchable cyan fluorescent protein for protein tracking," Nature Biotechnol., vol. 22, no. 11, pp. 1435-1439, 2004

[6] J. Boutet de Monvel, S. Le Calvez, and M. Ulfendahl, "Image restoration for confocal microscopy: Improving the limits of deconvolution, with application to the visualization of the mammalian hearing organ," Biophys. J., vol. 80, no. 5, pp. 2455-2470, 2001.

[7] W. Denk, J.H. Strickler, and W.W. Webb, "Two-photon laser scanning fluorescence microscopy," Science, vol. 248, no. 4951, pp. 73-76, 1990.

[8] B. Forster, D. Van De Ville, J. Berent, D. Sage, and M. Unser, "Complex wavelets for extended depth-of-field: A new method for the fusion of multichannel microscopy images," Microscop. Res. Tech., vol. 65, no. 1-2, pp. 33-42, 2004.

[9] S.F. Gibson and F. Lanni, "Experimental test of an analytical model of aberration in an oil-immersion objective lens used in three-dimensional light microscopy," J. Opt. Soc. Amer. A, vol. 8, no. 10, pp. 1601-1613, 1991.

[10] B.A. Griffin, S.R. Adams, and R.Y. Tsien, "Specific covalent labeling of recombinant protein molecules inside live cells," Science, vol. 281, no. 5374, pp. 269-272, 1998.

[11] M.G.L. Gustafsson, D.A. Agard, and J.W. Sedat, "I ${ }^{5} \mathrm{M}$ : 3D widefield light microscopy with better than $100 \mathrm{~nm}$ axial resolution," J. Microsc., vol. 195, no. 1 , pp. 10-16, 1999.

[12] R. Heintzmann, T.M. Jovin, and C. Cremer, "Saturated patterned excitation microscopy (SPEM)-A novel concept for optical resolution improvement," J. Opt. Soc. Amer. A, vol. 19, no. 8, pp. 1599-1609, 2002.

[13] S.W. Hell and E.H.K. Stelzer, "Fundamental improvement of resolution with a 4pi-confocal fluorescence microscope using two-photon excitation," Optics Commun., vol. 93, no. 5-6, pp. 277-282, 1992.

[14] S.W. Hell and J. Wichmann, "Breaking the diffraction resolution limit by stimulated emission: Stimulated-emission-depletion fluorescence microscopy," Optics Lett., vol. 19, no. 11, pp. 780-782, 1994.

[15] J. Huisken, J. Swoger, F. Del Bene, J. Wittbrodt, and E.H. Stelzer, "Optical sectioning deep inside live embryos by selective plane illumination microscopy," Science, vol. 305, no. 5686, pp. 1007-1009, 2004.

[16] M. Jacob and M. Unser, "Design of steerable filters for feature detection using Canny-like criteria," IEEE Trans. Pattern Anal. Machine Intell., vol. 26, no. 8, pp. 1007-1019, 2004.

[17] M. Liebling, A.S. Forouhar, M. Gharib, S.E. Fraser, and M.E. Dickinson, "4-dimensional cardiac imaging in living embryos via post-acquisition synchronization of nongated slice-sequences," J. Biomed. Optics, vol. 10, no. 5, 2005.

[18] J. Lippincott-Schwartz and G.H. Patterson, "Development and use of fluores- cent protein markers in living cells," Science, vol. 300, no. 5616, pp. 87-91, 2003. [19] J.S. Marchant, G.E. Stutzmann, M.A. Leissring, F.M. LaFerla, and I. Parker, "Multiphoton-evoked color change of DsRed as an optical highlighter for cellular and subcellular labeling," Nature Biotechnol., vol. 19, no. 7, pp. 645-649, 2001.

[20] L. McMahon, R. Legouis, J.-L. Vonesch, and M. Labouesse, "Assembly of C. elegans apical junctions involves positioning and compaction by LET-413 and protein aggregation by the MAGUK protein DLG-1," J. Cell Science, vol. 114, no. 12 , pp. $2265-2277,2001$.

[21] E. Meijering, M. Jacob, J.-C.F. Sarria, P. Steiner, H. Hirling, and M. Unser, "Design and validation of a tool for neurite tracing and analysis in fluorescence microscopy images," Cytometry Part A, vol. 58A, no. 2, pp. 167-176, 2004.

[22] X. Michalet, F.F. Pinaud, L.A. Bentolila, J.M. Tsay, S. Doose, J.J. Li, G. Sundaresan, A.M. Wu, S.S. Gambhir, and S. Weiss, "Quantum dots for live cells, in vivo imaging, and diagnostics," Science, vol. 307, no. 5709, pp. 538-544, 2005.

[23] M. Minsky, "Memoir on inventing the confocal scanning microscope," Scann., vol. 10, no. 4, pp. 128-138, 1988.

[24] J. Mitić, T. Anhut, M. Meier, M. Ducros, A. Serov, and T. Lasser, "Optical sectioning in wide-field microscopy obtained by dynamic structured light illumination and detection based on a smart pixel detector array," Optics Lett., vol. 28, no. 9, pp. 698-700, 2003.

[25] A. Miyawaki, A. Sawano, and T. Kogure, "Lighting up cells: Labelling proteins with fluorophores," Nature Cell Biol., vol. 5, pp. S1-S7, 2003.

[26] P.R. Norton, Handbook of Optics, vol. 1. New York: McGraw-Hill, 1995, ch. "Photodetectors," pp. 15.3-15.100.

[27] J.-C. Olivo-Marin, "Extraction of spots in biological images using multiscale products," Pattern Recognit., vol. 35, no. 9, pp. 1989-1996, 2002.

[28] G.H. Patterson and J. Lippincott-Schwartz, "A photoactivatable GFP for selective photolabeling of proteins and cells," Science, vol. 297, no. 5588, pp. 1873-1877, 2002.

[29] M. Petran, M. Hadravsky, M.D. Egger, and R. Galambos, "Tandem-scanning reflected-light microscope," J. Opt. Soc. Amer., vol. 58, no. 5, pp. 661-664, 1968.

[30] D.C. Prasher, V.K. Eckenrode, W.W. Ward, F.G. Prendergast, and M.J. Cormier, "Primary structure of the Aequorea victoria green-fluorescent protein," Gene, vol. 111, no. 2, pp. 229-233, 1992.

[31] C. Preza and J.A. Conchello, "Depth-variant maximum-likelihood restoration for three-dimensional fluorescence microscopy," J. Opt. Soc. Amer. A, vol. 21, no. 9, pp. 1593-1601, 2004.

[32] D. Sage, F.R. Neumann, F. Hediger, S.M. Gasser, and M. Unser, "Automatic tracking of individual fluorescence particles: Application to the study of chromosome dynamics," IEEE Trans. Image Processing, vol. 14, no. 9, pp. 1372-1383, Sept. 2005.

[33] O. Shimomura, "The discovery of aequorin and green fluorescent protein," $J$. Microsc., vol. 217, no. 1, pp. 3-15, 2005.

[34] C.ó. Sánchez Sorzano, P. Thévenaz, and M. Unser, "Elastic registration of biological images using vector-spline regularization," IEEE Trans. Biomed. Eng., vol. 52 , no. 4, pp. 652-663, 2005 .

[35] T. Stearns, "Green fluorescent protein. the green revolution," Current Biol., vol. 5, no. 3, pp. 262-264, 1995.

[36] D. Thomann, J. Dorn, P.K. Sorger, and G. Danuser, "Automatic fluorescent tag localization II: Improvement in super-resolution by relative tracking," J. Microsc., vol. 211, no. 3, pp. 230-248, 2003.

[37] H. Tsurui, H. Nishimura, S. Hattori, S. Hirose, K. Okumura, and T. Shirai, "Seven-color fluorescence imaging of tissue samples based on Fourier spectroscopy and singular value decomposition," J. Histochem. Cytochem., vol. 48, no. 5, pp. 653-662, 2000.

[38] J. Zhang, R.E. Campbell, A.Y. Ting, and R.Y. Tsien, "Creating new fluorescent probes for cell biology," Nature Rev. Molec. Cell Biol., vol. 3, no. 12, pp. 906-918, 2002.

[39] C. Zimmer, E. Labruyere, V. Meas-Yedid, N. Guillen, and J.-C. Olivo-Marin, "Segmentation and tracking of migrating cells in videomicroscopy with parametric active contours: A tool for cell-based drug testing," IEEE Trans. Med. Imag., vol. 21, no. 10, pp. 1212-1221, 2002.

[40] T. Zimmermann, J. Rietdorf, and R. Pepperkok, "Spectral imaging and its applications in live cell microscopy," FEBS Lett., vol. 546, no. 1, pp. 87-92, 2003.

[41] P. Sarder and A. Nehorai, "Deconvolution methods for 3-D fluorescence microscopy images," IEEE Signal Processing Mag., vol. 23, no. 3, pp. 32-45, May 2006.

[42] E. Meijering, I. Smal, and G. Danuser, "Tracking in molecular bioimaging," IEEE Signal Processing Mag., vol. 23, no. 3, pp. 46-53, May 2006.

[43] C. Zimmer, B. Zhang, A. Dufour, A. Thebaud, S. Berlemont, V. Meas-Yedid, and J.-C. Olivo Marin, "On the Digital Trail of Mobile Cells," IEEE Signal Processing Mag., vol. 23, no. 3, pp. 54-62, May 2006.

[44] X. Zhou and S.T.C. Wong, "Informatics challenges of high-throughput cellular and molecular miicroscopy," IEEE Signal Processing Mag., vol. 23, no. 3, pp. 63-72, May 2006. 